\title{
Los "útiles" para enseñar/aprender Lenguas en el Renacimiento y la IANUA LINGUARUM de W. Bathe (1611)
}

\author{
JAVIER Suso LÓPEZ \\ Universidad de Granada
}

Recibido: 7 febrero 2014 / Aceptado: 9 noviembre 2014

ISSN: $1697-7467$

\begin{abstract}
RESUMEN: Este artículo clasifica los útiles utilizados en la enseñanza/aprendizaje de las lenguas (lenguas clásicas, lenguas vulgares) en el Renacimiento, en los diferentes países europeos, en tres tipos: obras de índole gramatical; vocabularios; diálogos. En dicho contexto, resalta la novedad de las sentencias de la Ianua Linguarum (1611) como útil para adentrarse en la lengua.

Palabras clave: Gramática, útil gramatical, vocabulario, léxico, diálogo, coloquio, sentencia, enseñanza de lenguas, camino regular, camino irregular, camino medio, Ianua Linguarum, Renacimiento
\end{abstract}

The 'tools' to Teach/Learn Languages in the Renaissance and the IANUA LINGUARUM by W. BATHE (1611)

\begin{abstract}
This article classifies the language materials used in the teaching/learning of classic and vulgar languages in the Renaissance, in the different European countries, in three types: works of grammatical nature; vocabularies; dialogs. In this context, it appears that the sentences of the Ianua Linguarum (1611) are an usefully new gate to enter the language. Keywords: Grammar, lexicon, dialog, colloquium, sentence, modern language teaching, regular way, irregular way, Ianua Linguarum, Renaissance
\end{abstract}

\section{INTROdUCCIÓN}

Suele ser habitual, cuando se habla desde un punto de vista metodológico de la enseñanza de lenguas en el Renacimiento, señalar la existencia de

dos tendencias fundamentales, claras y contrapuestas [... que] pueden identificarse, precisamente, como gramatical, la una, y como conversacional, la otra. La primera está basada en la preeminencia de gramática; la segunda se fundamenta en la utilización prioritaria de materiales lingüísticos extractados de la realidad comunicativa (diálogos, frases) (Sánchez Pérez, 1992: 1-2).

En la misma obra, algo más adelante, opone la "especulación y análisis teórico" de la primera a la "práctica lingüística" de la segunda, si bien concluye: "no obstante, ambas perspectivas se 'conjugan' con frecuencia en mayor o menor grado" (ib., 1992: 24). 
Refiriéndose únicamente a las lenguas vivas, Titone expone por su parte que "il metodo d'apprendimento era essenzialmente pratico e non mutò per alcuni secoli. In quanto lingue vive, le lingue straniere andavano assorbite in maniera viva" (1985 [1980]): 24). Indica, no obstante, que tal método práctico era igualmente utilizado en las mejores escuelas en la enseñanza del latín (ib., 26). En un enfoque similar, Howatt (2004: 9-43) opone, refiriéndose a la Inglaterra de los siglos XV-XVI, el "practical language teaching" (en el caso de las lenguas vivas: Caxton, 1480; Bellot, 1588; Sainliens/Holyband, 1573 y 1576; Florio, $1578 \ldots$...) con una enseñanza gramaticalizada del latín (impartida en las grammar schools, precisamente, a partir de $A$ Short Introduction of Grammar, de Lily), destacando no obstante que surgen numerosas propuestas que conjugan uno y otro método, esencialmente para el latín (Ascham, 1570; Webbe, 1622...), pero que son transferidas posteriormente a las lenguas vivas. J. Caravolas incide en la misma oposición: "On divise d'habitude les méthodiciens des langues en deux groupes: ceux qui enseignent par 1' 'usage' et ceux qui enseignent par "règles"" (1994: 117), si bien añade: "Bathe (1611) y ajoute un troisième groupe où il classe les partisans du 'compromis', les éclectiques" (ib., 117). En un artículo algo posterior, Caravolas establece así tres modos de aprender lenguas (tanto muertas como vivas) en el Renacimiento: "par les règles", "par l'usage" y "par les règles et l'usage" (1995: 275-306). En efecto, Bathe titula el capítulo segundo de la Ianua Linguarum: "De tres caminos de aprender las lenguas, regular, irregular, y medio, y la comparación dellos entre sí" (1611: 30). Pretendemos en este artículo situar la obra de Bathe en el contexto histórico de los útiles existentes para la enseñanza de lenguas.

Hemos adoptado como metodología de trabajo partir de los útiles (el material pedagógico o los "language teaching materials") que confeccionaban los maestros y de los que disponían los "aprendices" de lenguas (frecuentemente, en situación de auto-aprendizaje), cuyo análisis nos permitirá inducir un modo de trabajar determinado. Dichos útiles constituyen en efecto otras tantas "puertas" o vías de acceso a la lengua: es decir, el tipo de útil empleado prefigura el acercamiento a realidades diversas de lengua, y por tanto, a modos diversos de enseñarla o aprenderla. Por razones de espacio, no nos adentramos en los mismos, reservando esta cuestión para un estudio posterior. De igual modo, utilizaremos el término método únicamente en el sentido de "modo de hacer" o de "camino" (que utiliza el propio Bathe), para marcar mejor la diferenciación entre el material pedagógico y el modo de hacer correspondiente. Pensamos finalmente, junto con Auroux (1994: 14), que debe enfocarse la historiografía lingüística no desde el "monumentalismo", sino, como indica Sáez Rivera, "tratar de entender los textos historiográficos desde la perspectiva de su época y cultura, al fin y al cabo el viejo y venerable método filológico" (2004: 796).

\section{LAS Diferentes "PUERTAS" PARA ADENTRARSE EN UNA Lengua}

Agrupamos los útiles en tres tipos:
A) unos útiles gramaticales
B) unos útiles léxicos
C) unos útiles conversacionales

Como veremos, las sentencias de la Ianua Linguarum (1611) constituyen un cuarto tipo de útil, totalmente novedoso. 
Los "útiles" para enseñar/aprender lenguas en el renacimiento...

\subsection{Los útiles gramaticales}

En el caso de las lenguas vivas, dichos útiles se elaboran según el modelo del Ars minor y el Ars maior de Donato ${ }^{1}$. Debemos considerar igualmente que las descripciones gramaticales (o "gramatización", Auroux, 1994) de las lenguas vernáculas vienen precedidas del uso de traducciones, en la lengua materna del "escolar", de fragmentos de gramática latina (en los inicios del aprendizaje, por tanto del Ars minor). Si bien esta tradición existe en diferentes países europeos (Inglaterra, Italia, Francia y España, fundamentalmente), el caso de Inglaterra es especialmente revelador, al presentar su población una situación de diglosia fuertemente marcada (francés-sajón, véase Ferguson, 1959), a la vez que un declive paulatino del francés entre la población noble. Ello obligaba a los maestros de lenguas a reforzar la enseñanza del francés en los inicios de los escolares en los rudimentos del latín. Como indica J. Barton en su prefacio al Donait François (1409): "les bones gens du roiaume d'Engleterre sont enbraséz a sçavoir lire et escrire, entendre et parler droit François" (in Colombo Timelli, 2012: 101). Encontramos así en Inglaterra una enorme variedad de útiles gramaticales en francés o en un latín sencillo, especialmente adaptados a dicha situación (además del referido Donait François), tales como:

- Le Traité ke moun sire Gauter de Bibelesworthefist a madame Dyonisie de Mounchensie pur apprise de language (hacia 1240)

- Femina ou Femina Nova (hacia 1415)

- el Tractatus Orthographiae y la Orthographia Gallica (finales del s. XIII), elTractatus orthographiae gallicane (finales del XIV)...

En otros países europeos existen igualmente estos esbozos de "gramáticas" en lengua vernácula: en Francia (véase las Grammaires de Metz, edición de Stadtler, 1964); en España y en Italia igualmente ${ }^{2}$. Las variaciones existentes en la factura del útil gramatical son por todo ello sumamente importantes, y sería erróneo inducir un único modo de utilización didáctica del mismo. Si repasamos los títulos que los autores adoptan para referirse a su obra, encontramos que puede tratarse de: introducciones (Nebrija, 1481 y 1488 ; Saulnier, 1607), esclarecimiento (Palsgrave, 1530), instrucción (Meurier, 1558), institución (anónimo de Lovaina, 1555) rudimentos, arte, camino ("acheminent" : Exact et très facile acheminement de la langue française, Pallet, 1606), método (La parfaicte méthode..., Charpentier, 1597), tratado (Meurier, 1553), reglas gramaticales (Corro, 1586), y, claro, gramática (Nebrija, 1492; Meurier, 1557). Adoptan el formato de obras gramaticales completas (simplificadas en diversos grados, según los criterios de la época; véase la Gramática de Nebrija, 1492), o bien limitan la descripción a algunas "partes" de la gramática: es el caso de las Conjugaisons, Regles et Instructions (Meurier, 1558), o bien de los numerosos tratados de ortografía y pronunciación ${ }^{3}$.

\footnotetext{
${ }^{1}$ Gramático latino del siglo IV. Su obra, Ars Gramatica, dividida en dos volúmenes (Ars minor y Ars maior) según el grado de dificultad, se convertirá en la gramática de referencia para la enseñanza del latín a lo largo de la Edad Media.

${ }^{2}$ Remitimos al artículo de M. Colombo Timelli (1998), que contiene una abundante bibliografía al respecto.

${ }^{3}$ Remitimos, para las gramáticas en lengua española, a la Bibliografía (BICRES I), establecida por Niederehe, 1995.
} 
Puede observarse que las obrasemplean como lengua vehicular tanto la lengua materna del eventual interesado (véase Palsgrave, 1530), como la lengua meta (Nebrija, 1492: libro V; anónimo de Lovaina, 1559), o bien ambas, en el caso de presentaciones bilingües (Meurier, 1558; anónimo de Lovaina, 1555; Corro, 1586) ${ }^{4}$. Para mayor abundamiento, son frecuentes las gramáticas de lenguas vivas redactadas en latín (véase: Isagogue, Sylvius, 1530; o Gallicae linguae Institutio, Pillot, 1550), abriendo así el elenco de interesados a los lectores de varias nacionalidades, pero limitándolos a quienes han efectuado estudios latinos. Digamos finalmente que la Gramática de la lengua castellana de Nebrija (1492) es en realidad una obra doble: además de presentar la primera descripción completa de una lengua vernácula, se compone de dos partes: una, destinada para uso de los propios castellanoparlantes; la segunda para uso de los extranjeros (el libro V se titula precisamente "De las introduciones de la lengua castellana para los que de estraña lengua querran deprender" (1492: 115-140)).

Si bien es imposible por tanto reducir a un molde único la descripción "gramatical", podemos referir algunas características comunes de tal tipo de obra. Digamos así que, con dicho tipo de útil, la entrada a la lengua se efectúa comenzando por aprender sus elementos constitutivos más pequeños, e ir progresando según las diferentes combinaciones regladas de los mismos:

- la unidad mínima: cada una de las letras (con su "valor" o "fuerza": el sonido que representa), objeto de la Pronunciación y Ortografía;

- las combinaciones de las letras (las sílabas), objeto de la Prosodia;

- las combinaciones de las sílabas bajo forma de palabras, así como sus "accidentes": sufijos, prefijos, raíz, marcas de persona, número, género y tiempo, la concordancia entre palabras...; objeto de la Analogía o de la Etimología (morfología, en la denominación actual);

- las combinaciones de las palabras bajo forma de frases, objeto de la parte Construcción (Sintaxis).

Como indica Holtz (1981: 91-93), tal presentación de la lengua se efectúa mediante un procedimiento piramidal, que permite dividir el cursus de formación en tres niveles (básico: ortografía, en que se aprende a leer; elemental: morfología, y superior: sintaxis). El enfoque es nítidamente formal: la palabra interesa en cuanto entidad sujeta a su descomposición en partes o bien su reunión en oraciones; la significación no interesa, únicamente las posibles formas externas que toman las palabras.

\subsection{Los útiles léxicos: los glosarios (o catálogos de palabras clasificadas por temas)}

En este caso, se transpone a la lengua vernácula el modelo latino de los nominalia, que hunde sus raíces tanto en las Glosas (s.VIII-IX), como en el Vocabulario de Aelfric (siglo $\mathrm{X}$ ). La situación lingüística de Inglaterra, expuesta anteriormente, hace florecer igualmente la edición de innumerables catálogos léxicos bilingües (latín-francés; latín-sajón; sajón-francés), tales como Anglo-saxon vocabulary (s. XI), Semi-saxon vocabulary (s.XII), The Treatise de

\footnotetext{
${ }^{4}$ Remitimos a la Bibliografía temática [...] de Esparza Torres et al. (2008), para las referencias acerca la lengua española o bilingüe (español-francés).
} 
Utensilibus (Alexandre Neckam, s.XII), Dictionnarius (John de Garlande, primera mitad del s. XIII), Vocabulary of the Names of Plants (s. XIII), Names of the parts of the human body (de fecha desconocida), English vocabulary (s. XV), Nominale (s. XV); A pictorial vocabulary (s. XV). El Vocabula de Stanbridge (1507) contiene así "les parties du corps, leurs fonctions, les sens, les maladies, la parenté, la chambre, les meubles, les vêtements, la cuisine, les ustensiles, etc., en anglais et en latin" (Caravolas, 1994 : 87). Estos útiles se transfieren al aprendizaje del francés en Manières de langage (1396), en que el vocabulario está insertado en estructuras frásticas listas para ser utilizadas (véase más adelante). Por supuesto, en otros países europeos, fundamentalmente en zonas de frontera lingüística, como en Flandes, se desarrollan útiles equivalentes: así, un "maestro de la escuela de la ciudad de Brujas" compone un Livre des mestiers. Dialogues français-flamands (mediados del s. XIV), destinado a los mercaderes (véase reed. Michelant, 1875), en que ensambla una relación (prácticamente completa) de productos con fórmulas básicas utilizadas en toda compraventa. Tales útiles circulan de un país a otro: en la Tres bonne doctrine pour aprende briefment fransoys et engloys (hacia 1480), Caxton básicamente sustituye la columna del neerlandés por el inglés, recogiendo cerca de 1100 palabras. Dicha tradición da lugar al Vocabulair pour apprēdre romain et flameng (1496-1501, Amberes: Roger van der Dorpe), obra que será reimpresa por Willem Vorsterman, con el título de Vocabulario para aprender Franches Espannol y Flamincp (1520-1530), "primer manual para la enseñanza de la lengua española impreso fuera de la península", como indica Núñez (2007). Dicho filón prosigue con el Vocabulaire [...] françois et Flameng de Berlaimont (primera edición entre 1527 y 1530), que incorpora otras lenguas a lo largo de las sucesivas de los siglos XVI y XVII, fundiéndose con la tradición italiana representada por los Dilucidissimus dictionarius, Solenissimo vochabuolista, Utilissimo Vocabulista o Introito e porta, a partir de un primer vocabulario veneciano-alemán, de 1477, como ha expuesto Rossebastiano Bart (1984), constituyendo las fuentes básicas de la lexicografía monolingüe y bilingüe. No podemos olvidar en esta relación el Vocabolari molt profitos per apendre Lo Catalan Alemany y Lo Alemany Catalan, editado en Perpiñán, en 1502 (Johan Rosembach).

Ahora bien, si los vocabularios temáticos poseen una orientación práctica, y se dirigen a un público variado (mercaderes, viajeros, estudiantes), los diccionarios (ordenados por orden alfabético) ${ }^{5}$, igualmente bilingües o plurilingües (Calepinus, Nomenclator), se dirigen a un público culto (humanistas), y sus inicios deben mucho al Vocabulario latino-español (1492) y el Vocabulario español-latino (1495) de Nebrija.

Adoptamos la propuesta de Colombo Timelli (1998: 28-29) de diferenciarlos, denominando vocabularios "prácticos" a los primeros (ordenación temática), y diccionarios cultos a los segundos (ordenación alfabética) ${ }^{6}$. En ambos casos, el enfoque con el que se aprehenden las palabras es de tipo semántico: el discípulo debe relacionar cada palabra de una frase de la lengua extranjera con la palabra correspondiente en la lengua materna. Supone una operación mental de tipo onomasiológico, siendo un primer paso hacia la comprensión completa de una frase (traducción literal).

\footnotetext{
${ }^{5}$ Existentes ya en el medievo en algunas compilaciones (como el Catholicon de Balbi, 1280).

${ }^{6}$ Existen en todo caso útiles intermedios, como el Dictionnarium de Berlaimont (1527-30,1536..., véase más adelante)
} 
La importancia atribuida al útil léxico procede de una concepción de la lengua - propia de la antigüedad y del medievo, que se prolonga en el Renacimiento chocando con la nueva visión de los humanistas - , según la cual se efectúa una asimilación entre cosa $=$ palabra $=$ concepto. Si a cada objeto exterior (o bien cada noción abstracta) le corresponde una palabra (que representa a su vez el concepto), el modo de comprender una lengua extranjera (muerta o viva) consiste básicamente en reemplazar una palabra de la LE por la correspondiente en la L1, y a la inversa si queremos hablarla. El literalismo en la traducción es defendido así por San Jerónimo (finales del s. IV- principios s. V), Boecio (s. V) y Escoto Erigena (s. IX), entre otros. Escaligero (s. XV-XVI) dirá así: "words are the signs of things in the real world, and these signs in turn correspond to mental concepts. The concepts, like the phenomena in the world, are immanent and remain the same across different languages" (Padley, 1985: 243, in Hermans, en línea) ${ }^{7}$.

Terminamos este apartado con dos consideraciones complementarias. En primer lugar, la fraseología forma parte de este tipo de útiles (expresiones lexicalizadas con sentido propio, maneras de hablar, idiotismos, perífrasis, refranes...). En segundo lugar, la ordenación temática (o por "tópicos") de los términos recogidos en un glosario induce una utilización pedagógica del mismo, puesto que facilita tanto su memorización como su reutilización en los diálogos (véase apartado siguiente). Por su parte, la ordenación alfabética del léxico (bajo forma de "diccionario") facilita por su parte la búsqueda de un ítem determinado: esta disposición (analítica, semasiológica) induce por su parte una utilización diferente: la traducción, la lectura personal de textos complejos en contextos de autoaprendizaje... No debemos tomar los títulos de las obras de modo literal: por ejemplo, el Dictionarium quator linguarum (1556, Lovaina, Gravio) es en realidad un vocabulario temático, y contiene además un tratado acerca de las cuatro partes indeclinables (de la oración), un tratado de ortografía francesa, y un modo de escribir la lengua española.

\subsection{Los útiles conversacionales}

Estos útiles se enmarcan igualmente en una larga tradición ("la forme dialoguée est sans doute aussi vieille que l'institution scolaire", Holtz, 1981: 100), que remonta, en sus fuentes escritas, a los Hermeneumata Pseudo-Dositheana (principios del siglo III), diálogos que, con múltiples modificaciones, siguen utilizándose en la enseñanza del latín a lo largo del medievo (véanse los Coloquios de Aelfric, s. X, o el Liber Donati, de Kingsmill, s. XIV). Como indica Chevalier (1998: 18), este tipo de útil se extiende a la enseñanza del francés en la Inglaterra de los siglos XIV y XV, dada la situación de diglosia descrita anteriormente (nobleza, juristas, mercaderes, administradores y "lettrés"), así como en las zonas lingüísticas fronterizas del norte de Francia y Flandes, a través de varios tipos de realizaciones. En Inglaterra, se edita así la obra Manières de langage (editado en Inglaterra, 1396), que constituye un "model conversation book[s], intended for the use of travellers, merchants, and others desiring a conversational and practical rather than a thorough and grammatical knowledge of French" (Lambley, 1920: 35). La obra se estructura en forma de relato de un

\footnotetext{
${ }^{7} \mathrm{http}: / /$ www.ucl.ac.uk/cics/translation-in-history/Hermans_pdf [10.12.2011]

${ }^{8}$ Se conservan diversos manuscritos al respecto. Y no son el único útil de esta clase: Gessler (1934) detalla la existencia de Le Petit Livre (1399) y de Les Dialogues françoys (1415), entre otros.
} 
caballero que indica al lector las expresiones que éste puede utilizar en diferentes situaciones de la vida diaria, procedimiento que permite al autor recoger diversas formas frásticas equivalentes (en una pregunta o en una respuesta), así como presentar el vocabulario temático correspondiente. En Flandes, se edita el Livre des Mestiers (Brujas, mediados s. XIV), que contiene igualmente estructuras frásticas listas para ser utilizadas (en presentación bilingüe francés-neerlandés). Ambas propuestas se funden en los Dialogues in French and English (1480) y en el Book of travellers (hacia 1483), de Caxton, reemplazando el neerlandés por el inglés. Estos diálogos, al igual que A Lytell Treatise for to lerne Englishe and Frensshe (Wynken de Worde, 1498), adaptan los temas de "conversación" a los nuevos destinatarios, manteniendo en lo esencial los principios de composición anteriores. La deuda con respecto a tal tradición de la obra: Introduction. Very profitable book to learn English and Spanish (Alston, 1554), editada en ocasión del proyectado enlace entre la reina María y Felipe II, es por ello enorme: "It contains dialogues, a vocabulary, and some religious material. While there is everyday information in the manual, it also addresses 'the maner of byenge and sellyng [...] the ways of callynge upon your debtors' and 'the maner of writing epistles and letters of obligations, solutiõs, and of bargains" "(Corrigan, 2005: en linea) ${ }^{9}$. Por su parte, el French SchooleMaster (1573) y el French Littleton (1576) y de Sainliens/Holyband combinan diálogos (compuestos en la tradición de Manières de langage) y observaciones de tipo gramatical.

En el siglo XVI, como indica Colombo Timelli (1998: 28), los diálogos están habitualmente presentes en los vocabularios bilingües y/o plurilingües "prácticos", como complemento natural en vistas a la utilización didáctica de los mismos. Tanto es así que el Dictionarium de Berlaimont $(1527-30,1536,1556 \ldots)$, a partir de la edición de 1556 cambia el título por el de Colloquia et dictionariolum... Esta obra merece una consideración especial, como observa Colombo Timelli: reúne diálogos (4), modelos de cartas, un tratado de ortografía y de fonética, un tratado sobre conjugación, un listado de palabras ordenado alfabéticamente (solo en francés):

On aura reconnu sans peine la typologie des textes élaborés en Angleterre entre le XIV ${ }^{\mathrm{e}}$ et le $\mathrm{XV}^{\mathrm{e}}$ siècle pour l'enseignement du français : une 'manière de parler' (les dialogues), un 'ars dictaminis' (des lettres), un 'nominale' (lexique), un traité d'orthographe (1998: 30).

Si observamos la evolución de este tipo de útiles, podemos apreciar que no todos los diálogos son de tipo "funcional", al entrecruzarse con los coloquios humanistas -como los compuestos por Erasmo (Colloquia, Bâle, 1516) o Vives (Linguae latina exercitatio, 1539)-, que se reeditan rápidamente en versiones bilingües en las diferentes lenguas vernáculas. En efecto, entre los útiles para enseñar lenguas del siglo XVI, apreciamos la existencia de dos tipos de diálogos:

a) los diálogos de tipo "funcional" (o pragmático), en los que dos personajes ejercitan una lista de términos previamente expuesta, agrupados en torno a un tema determinado (nomenclatura, nominalia). Tales diálogos incluyen por tanto las expresiones (o "rutinas") correspondientes, propias de las diferentes situaciones de uso (o de "comunicación") de la vida diaria: para saludar, para comprar, de viaje, etc. Se trata

\footnotetext{
${ }^{9} \mathrm{http}: / /$ homes.chass.utoronto.ca/ cpercy/courses/6361 corrigan.htm [10.12.2013]
} 
de diálogos breves, pero suelen encadenarse unos temas con otros. Proponemos mantener el término "diálogos" para referirnos a los mismos. A modo de ejemplo, el Dictionarium quator linguarum (Lovaina, 1556) pone en escena las siguientes situaciones:

Le premier Chapitre est un convive à dix personnages, \& contient beaucoup de comunes devises, desquoy on use comunement a table, Le deuxieme Chapitre est de vendre $\&$ d'accepter. Le troisieme Chapitre est pour demander ses debtes. Le quatrieme Chapitre est pour apprendre à faire les lettres missives, obligations, quittances, \& contract de louage (1556: A iij)

Tal tipo de diálogos se desarrolla según una multiplicidad de situaciones (la edición de 1587 incluye siete lenguas, y contiene 7 diálogos; posteriormente, serán 10). Serán recogidos - con las variantes y adaptaciones al gusto de cada país y autor, como muestran Lépinette (1996) y Sáez Rivera (2004) -, por Meurier (Coloquios familiares [...], 1568), Sotomayor/Liaño (1565), Sainliens/Holyband (1578), Stepney (The Spanish schoolemaster, 1591), Minsheu (cuyo Dictionarie in Spanish and English... incluye unos Pleasant and delightful dialogues, 1599), C. Oudin (Diálogos en Español y Francés, 1604, insertados igualmente en su Gramática de 1606), Saulnier (Introduction en la langue Espagnolle [...], 1608), etc.

b) Los diálogos de tipo "conversacional”, en los que dos o más personajes se entretienen charlando acerca de "lo divino y de lo humano". Se trata de diálogos más largos, y gozan de mayor libertad de composición. Utilizaremos el término "coloquios" para referirnos a los mismos. Así, sin entrar en detallar las características de estos coloquios (por falta de espacio), si observamos el Vocabulario de Sotomayor/Liaño (1565: 66-88), podemos apreciar que cuatro diálogos poseen un carácter práctico o funcional, mientras que otros cuatro poseen un carácter literario o culto según los temas apreciados en las reuniones galantes de la época: "Dialogue et propoz amoreux de troys bergiers, et une bergiere [...] (70-80), quienes discuten sobre la enfermedad del amor; la "Plainte de Silvio pour estre absent de sa bergere Laurie par Ile", composición de tipo poético $(80-81)^{10}$; una "Lettre d'ung amy a aultre, l'ung demandant qu'il luy face sçavoir en quel temps se perdist Espaigne" (83-85), de temática histórica; y "Propos de deux amys [...]" (85-88), en la que discuten acerca de cuál es el órgano más peligroso del cuerpo humano ${ }^{11}$.

La razón que preside la elección de tal tipo de diálogos reside en las características de sus destinatarios: la corte de gentilhombres y damas de compañía que acompañan a Isabel de Valois tras su boda con Felipe II (1559), quienes desean integrarse en los usos y costumbres de la corte española. Este tipo de útil dará paso en el siglo XVII a la utilización didáctica de obras literarias: no solo en formato de diálogos (de carácter culto o sabio), sino también de textos de tipo literario, histórico, religioso,

\footnotetext{
${ }^{10}$ El título de la obra menciona la existencia igualmente de una "égloga", que no puede ser sino el diálogo ${ }^{\circ}$ 4, dirigido a la amada (ausente).

${ }^{11}$ Tras examinar los méritos respectivos del corazón, la carne, los ojos, concluyen que es la lengua, pues es ¡el único órgano que puede producir la muerte! Y por tanto, es preciso prevenirse de las malas lenguas. Citamos a partir de la reedición de Corcuera\& Gaspar (1999).
} 
así como de composiciones poéticas (agrupadas en florilegios), y frecuentemente en versiones bilingües.

El uso de la presentación dialogada era frecuente en la época, incluso en obras destinadas a personas cultas. Pensemos por ejemplo en los Diálogos sobre la lengua, de Juan de Valdés (1535), obra destinada a mejorar el conocimiento del español por parte de nativos (elegancia, propiedad) y de extranjeros (italianos, en este caso). Corro pensaba completar sus Reglas gramaticales (1588) con el Diálogo en que particularmente se tratan las cosas acaecidas en Roma en el año de 1527, oponiéndose el editor a ello ${ }^{12}$. Tales útiles serán utilizados en los siglos XVII y XVIII en contextos avanzados de aprendizaje, o bien con discípulos pertenecientes a la nobleza, quienes deseaban brillar en sociedad, como es el caso de la Gramática francesa con un arte poética y [...] un compendio de la poesía francesa, de Billet, compuesta en 1673, y reeditada en 1688 y 1708.

En cualquier caso, los diálogos (y de modo más claro, los coloquios cultos) parten de una concepción de la lengua que se extiende en el Renacimiento, manifestada por la traducción de Erasmo del Nuevo Testamento, en 1516: el texto bilingüe (griegolatín) se acompaña de innumerables notas a pie de página, en las que el autor interpreta o justifica su elección. Así, a veces hace corresponder el $\lambda o ́ \gamma o \varsigma$ griego con el términoverbum (palabra) y a veces con sermo (discurso). Se abre paso así a un desmantelamiento de la traducción literal, y por ende, de la correspondencia palabra-concepto sobre el que se basa, surgiendo una nueva concepción de la lengua. Los humanistas (véase Dolet, Manière de bien traduire d'une langue à une autre, 1540) defienden que cada lengua representa la realidad exterior de modo particular, y que posee por tanto un vocabulario específico (que no se corresponde sino parcialmente con otras lenguas), así como una disposición propia de las palabras en frases (constructio). La traducción literal ("mot-à-mot") es por tanto un contrasentido: para reproducir fielmente el pensamiento del autor original, es preciso reorganizar su discurso (palabras, construcción) en la lengua meta fundándose tanto en los conocimientos lingüísticos como en la cultura de la época (de ahí, la Filología). Ello se traduce, en el caso del aprendizaje de una lengua, por el abandono (relativo) del útil léxico y el reforzamiento del útil conversacional, discursivo y textual.

\subsection{Las sentencias de la Ianua Linguarum (1611)}

En el contexto esbozado en los puntos anteriores, la Ianua Linguarum (1611) "compuest[a] por ciertos padres irlandeses del colegio de Salamanca” (1611: Tassa y Aprobación), en la que destaca la labor personal del irlandés William Bathe, "après vingt ans de labeur avec ses collaborateurs" (Caravolas, 1994: 110), no puede sino ser considerada como un útil totalmente novedoso. Así lo entendieron varios profesores de lenguas de la época, que reciben con admiración y gozo la publicación de dicha obra $(1611: 2,4,5)$.

\footnotetext{
${ }^{12}$ Esta obra se conoce como Diálogo de Lactancio y un Arcediano: "en él, a la luz de las ideas erasmistas, hace Valdés una defensa del emperador Carlos V con motivo del saqueo de Roma por las tropas imperiales en 1527" (Nieto, 1988: 5, nota 2).
} 
El éxito de la obra fue en efecto fulgurante: tres años más tarde, se edita una edición en la que se añade el portugués; en 1615, es editada una Ianua en Inglaterra con la traducción al inglés de las sentencias; en los años sucesivos, se editan versiones plurilingües, añadiéndose lenguas sucesivamente (6 lenguas en una edición de 1629). El propio Comenius edita una Ianua linguarum reserata (1631), mejorándola en algunos aspectos, que popularizará este tipo de obra en toda Europa ${ }^{13}$.

La Ianua Linguarum está precedida, en versión bilingüe latín-español como el resto de la obra, por un Proemio (1611: 25-27) y diez capítulos (28-39), en los que los autores explican, entre otras cosas, la composición de la obra, los fundamentos metodológicos ("de tres caminos", "del camino medio") y el orden que ha de seguirse para esta "obrezilla". Dichos capítulos constituyen el primer tratado de didáctica de las lenguas, y constituyen el precedente más notable del Novissima Linguarum Methodus de Comenius (1648).

La Ianua Linguarum se compone de doce centenas de frases (42-130) —disponiéndose las frases latinas en las páginas pares y la traducción correspondiente en español en las páginas impares-, de un apéndice de términos equívocos (132-139), y de un listado de los términos latinos utilizados en las centenas ordenados por orden alfabético con indicación de la centena en que son utilizados (141-215). El Proemio resalta los destinatarios que se beneficiarán con dicha obra (varones apostólicos, confesores, gramáticos y retóricos, preceptores, caminantes, embajadores, criados, nobles...), en el aprendizaje de las lenguas muertas, pero igualmente de las lenguas vulgares (como resalta el título de la obra: ad omnes linguas intelligendas).

Frente a los anteriores catálogos de sentencias morales, los autores destacan que en las sentencias por ellos compuestas no se repite ninguna palabra y que están juntadas en un orden muy estudiado. Este útil mejora a su juicio el contacto práctico con la lengua que se obtiene viajando al país, mejorando la efectividad y ganando mucho tiempo:

Por que asi como fuera mucho mas fácil conocer por vista de ojos todos los animales, visitando el arca de Noe, que contenia de cada genero dos escogidos, que no andando todo el mundo hasta que acaso topasse un animal de cada genero: de la misma manera se aprenderâ todos los vocablos muchos más fácilmente con el uso destas sentencias, donde se contiene los fundamentos de todos, que sin algunas reglas, oyendo, hablando, o leyendo, hasta que acaso alguno topeçó tantos vocablos (1611: 27).

En la Ianua se mantiene una concepción de la lengua tradicional: la lengua es un instrumento por el que se designan las cosas existentes en el mundo; aprender una lengua consiste en primer lugar en aprender el léxico; en la medida en que las sentencias agrupan los vocablos de modo ordenado y sistemático, y se proporciona su traducción a la vista, se facilita su aprendizaje. El valor de esta "obrezilla", según destacan los autores, consiste así en racionalizar la disposición del vocabulario (en relación con los Vocabularios prácticos como con los Diccionarios alfabéticos), eliminando "muchas palabras raras" (poco frecuentes), resaltando el parentesco formal entre palabras (derivación, composición, prefijos, sufijos), e insertando las mismas en sentencias fáciles de retener. El orden de las sentencias refleja a

\footnotetext{
${ }^{13}$ Véase al respecto Caravolas 1994: 339-369.
} 
su vez el orden (la arquitectura) de la naturaleza, aprehendida y clasificada racionalmente. De ahí deriva su valor didáctico: al insertarse los vocablos en frases, su memorización se ve fuertemente facilitada: "las palabras puestas en el Vocabulario ningún sentido tienen: de donde nace que la memoria desamparada del ayuda del entendimiento no los retiene" (1611: 30).

En segundo lugar, es preciso destacar que el aprendizaje de los vocablos (a través las sentencias) no constituye sino la primera etapa del aprendizaje de una lengua, que continuará posteriormente con la gramática (concordancia) y con los autores, como se indica en el capítulo primero:

El cuerpo de qualquiera lengua consta de quatro miembros principales, de vocablos, concordancias, phrases, y elegancia: los vocablos da el Diccionario, las concordancias la Gramatica, las phrases los autores, la elegancia pìntan los Rhetoricos con sus figuras: llamamos phrases las propiedades, o particulares modos de hablar de cada lengua (1611: 29-30).

Tras aprender los vocablos, es preciso aprender las concordancias, y posteriormente las "phrases" y las figuras. El "camino medio" de la Ianua consiste así ante todo en un nuevo ordenamiento del aprendizaje lingüístico, mejorándolo además mediante una presentación igualmente novedosa.

\section{LOS "CAMINOS" DE ENSEÑANZA/APRENDIZAJE DE LENGUAS EN EL RENA- CIMIENTO}

Es preciso señalar, en primer lugar, que los diferentes "modos de hacer" expuestos en la Introducción suponen una abstracción con respecto a lo realmente practicado en los diversos contextos de enseñanza/aprendizaje (escolares, preceptorado, autoaprendizaje, adultos: mercaderes, soldados, hombres de palacio, viajeros, mensajeros e intérpretes, véase Dictionarium quator linguarum, 1556: Al lector). El examen de los útiles existentes nos hace ver que los autores agrupan en ellos componentes diversos (reglas de "pronunciación", paradigmas morfológicos, vocabulario, diálogos), en proporciones diversas, en función de dichos contextos y de los objetivos que perseguía cada uno de los interesados en aprender una lengua.

En segundo lugar, estos "modos de hacer" no poseían la fuerza de un modelo teórico ("método" en Galisson\&Coste, 1976; "metodología", en Puren, 1988: 17), sino que se practicaban con una enorme flexibilidad, en función de los útiles de los que se disponía y de los contextos de enseñanza/aprendizaje descritos. Es posible, sin embargo, reagrupar un conjunto de acciones, derivadas de un determinado tipo de útil, e intentar dotarlas de un sentido común a las mismas. De ahí que pueda proponerse la existencia de tres caminos: regular, irregular y medio (por parte de los autores de la Ianua Linguarum) en una aprehensión abstracta que ayuda a entender los posicionamientos y las tendencias metodológicas de la época.

En dicho contexto, debemos decir que la Ianua Linguarum no pretende ningún tipo de eclecticismo. Es decir, no reúne el camino regular (el aprendizaje de las reglas de concordancia) y el camino irregular (el "común uso de la lengua") en un camino, intermedio, que sería el resultado de una simbiosis de los dos anteriores. En todo caso, como dicen los 
autores, reúne la certeza del primero con la facilidad del segundo. Con dicho útil, los interesados en aprender una lengua no aprenden reglas (para posteriormente recitarlas al maestro) ni memorizan diálogos prácticos similares a los que encontrarán en su vida diaria. Las sentencias permiten a los "curiosos" únicamente aprender el vocabulario de modo ordenado (o 'regular'), comprendiendo su significación gracias a la sentencia en que está insertado y facilitando su memorización. Las sentencias no están pensadas para ser reutilizadas en una situación de uso "real". En una fase posterior, la persona interesada emprenderá el estudio de las restantes partes de la lengua: la gramática (concordancia), las frases y/o la elegancia según sus deseos de proseguir el aprendizaje. El texto de la Ianua no deja lugar a dudas:

Para aprender las lenguas, que constan de las cuatro partes dichas, hasta agora tan solamente se han hallado dos caminos: el regular, qual es, para guardar las concordancias la Gramatica, y el irregular, qual es, el comun uso de los que aprenden por lección, o habla en las lenguas vulgares. [...] (1611: 30)

\section{A MOdo de CONClusión}

La presentación que hemos esbozado (pues se necesitaría profundizar la cuestión de los modos de hacer), nos permite avanzar varias ideas: la abundancia de útiles que se ponen a disposición de los interesados en aprender lenguas; la imposibilidad de tipificarlos de modo cerrado; la flexibilidad que inducen por ello en los modos de hacer en la enseñanza/aprendizaje de las lenguas vivas en el Renacimiento. En dicho contexto, la Ianua Linguarum surge como una puerta de entrada a la lengua novedosa a la vez que ingeniosa, que atraerá la atención de muchos maestros y aprendices de lenguas, acompañada de una primera reflexión acerca de la didáctica de las lenguas.

\section{REFERENCIAS BIBLIOGRÁFICAS}

\section{Fuentes primarias ${ }^{14}$}

Ascham, R. (1570). The Scholemaster [...]. Londres: John Day.

Bathe, W. (1611). Ianua linguarum, sive modus maxime accommodatus, quo patefit aditus ad omnes linguas intelligendas [...]. Salamanticae: apud Franciscum de Cea Tesa.

Bellot, J. (1588). The French method [...]. Londres: Robert Robinson.

Caxton, W. (1480). Dialogues in French and English. Londres: Caxton. Reed. éd. Bradley, 1900, Early Text Society, Londres: Kegan Paul, Trenh and Tribuner.

Corro, A. del ([1588] 1988). Reglas Gramaticales para aprender la Lengua Española y Francesa, confiriendo la una con la otra, segun el orden de las partes de la oration Latinas. Oxford, Joseph Barnes. Reed. de Lidio Nieto Jiménez, Madrid: Arco Libros. Dictionnarium quator linguarum [...]. 1556. Lovaina, Gravio.

\footnotetext{
${ }^{14}$ Referimos únicamente las fuentes primarias que han dado lugar a alguna cita; para las demás, remitimos a las fuentes secundarias en las que tales obras son catalogadas y/o estudiadas, así como a Niederehe, H.J. (1995).
} 
Du Wes, G. (hacia 1532). An introductorie for to lerne to rede, to pronounce and to speke French trewly [...]. Londres: Thomas Godfray.

Florio, J. (1578). His First Fruites [...]. Also a perfect Inductio to the Italian, and English tongues. Londres: Thomas Dawson.

La manière de langage qui enseigne à parler et à écrire le français, publ. según el MS. du Mus. Brit. Harl. 3988 [by M.P.H. Meyer, 1873].

Le Livre des Mestiers: Dialogues français-flamands composés au XIVe siècle par un maître d'école de la ville de Bruges (1875 [s. XIV]). Paris: Librairie Tross, ed. H, Michelant. Reed. J. Gessler (ed.): Le Livre des mestiers de Bruges et ses dérivés : quatre anciens manuels de conversation. Brujas: Maitres imprimeurs brugeois; Fondation Universitaire de Belgique, 1931. 6 v.

Meurier, G. ([1557] 2005). La grammaire Françoise, contenante plusieurs belles regles propres \& necessaires pour ceulx qui desirent apprendre la dicte langue, Anvers, Plantin. Reed. A cargo de Colette Demazière, Paris : Honoré Champion.

-([1558] 1973). Conjugaisons, Regles et instructions, mout propres et necessairement requises, pour ceux qui désirent apprendre François, Italien, Espagnol et Flamen : dont la plupart est mise par maniere d'Interrogations et Responses. Amberes: J. van Waesberge.

Sotomayor, B. de (1565). Grammatica con reglas muy prouechosas y necessarias para aprender a leer y escriuir la lengua Francesa conferida con la Castellana, con vn vocabulario copioso de las mesmas lenguas [...]. Impressa en Alcala de Henares: en casa de Pedro de Robles y Francisco de Cormellas. Contiene el Vocabulario de los vocablos de Jacques de Liaño (o Ledel).

Valdés, J. de (1535). Diálogo de la lengua, Madrid. Reed. Buenos Aires: Ed. Sopena, 1940; José F. Montesinos, Madrid: Espasa-Calpe, 1976; Madrid: Olympia ediciones, 1995.

Valence, P. (1528). Introductions in Frensche [...]. Londres: Wynken de Worde.

Webbe, J. (1622). An Appeale to Truth. In the Controversie between Art and Use ; about the best, and most expedient Course in Languages. To be read Fasting. Londres: H.L. for George Latham.

\section{Fuentes secundarias}

Auroux, S. (1994). La révolution technologique de la grammatisation. Bruxelles: Mardaga.

Caravolas, Jean (1994). La didactique des langues. Précis d'histoire I (1450-1700), MontréalTübingen: Presses de l'Université de Montréal-Gunter NarrVerlag.

_ (1995). "Apprendre une langue étrangère à la Renaissance", in Historiographia linguistica XXII, 3, 275-307.

Chevalier, J.-Cl. (1988). "Les dialogues médiévaux. Origines, filiations, méthodes. Quelques pistes", in Documents pour l'enseignement du français langue étrangère ou seconde, 22, 17-26.

Colombo Timelli, M. (1998). "Dialogues et phraséologie dans quelques dictionnaires plurilingues du XVI siècle", in Documents pour l'enseignement du français langue étrangère ou seconde, 22, 27-59.

- (2012). "Ruptures ou continuité? La grammaire de matrice donatienne au siècle de la Renaissance", in B. Colombat et al., Vers une histoire générale de la grammaire française [...], Paris: H. Champion, 97-114.

Corcuera, F. y Gaspar, A. (1999). La lengua francesa en España en el siglo XVI [...]. Zaragoza: PU. Corrigan, A.J. (2005). Manuals for Teaching English as a Foreign Language in the 15th and 16th Centuries. http://homes.chass.utoronto.ca/ cpercy/courses/6361 corrigan.htm [10.12.2013] 
Esparza Torres, M.A. et al. (2008). Bibliografía temática de historiografía lingüistica española: Fuentes secundarias, 2 Vol. Hamburgo: BuskeVerlag.

Ferguson, Ch. (1959). "Diglossia", in Word, 15, 325-340.

Hermans, T. (s.d.). Etienne Dolet. The Way to Translate Well from One Language into Another. Http://www.ucl.ac.uk/cics/translation-in-history/Hermans_pdf [10.12.2011]

Galisson, R. ; Coste, D. (1976). Dictionnaire de didactique des langues. Paris: Hachette.

Holtz, L. (1981). Donat et la tradition de l'enseignement grammatical, étude et édition critique. Paris, CNRS.

Howatt, A.P.R. (2004 [1984). A History of English Language Teaching.Oxford: OUP.

Kibbee, Douglas A. (1989). "L'enseignement du français en Angleterre au XVI siècle", in P. Swiggers y W. Van Hoecke, La langue française au XVIe siècle: usage, enseignement et approches descriptives. Leuven:UniversityPress, 54-77.

Lambley, K. (1920). The Teaching and Cultivation of the French Language in England During Tudor and Stuart Times. Manchester: Manchester UP.

Lépinette, B. (1996). "Les premières grammaires du français (1565-1799) publiées en Espagne. Modèles, sources et rôle de l'espagnol”, in Histoire, Épistémologie, Langage 18/II, 149-177.

Niederehe, H.J. (1995). Bibliografía cronológica de la lingüistica, la gramática y la lexicografía del español (BICRES I): desde los comienzos hasta el año 1600. Amsterdam, [etc.]: John Benjamins.

Nieto, L. (1988). "Estudio introductorio". Reglas gramaticales para aprender la lengua española $y$ francesa. Madrid: Arco Libros.

Núñez, L.P. (2007). "Un ejemplar raro en la Biblioteca Histórica : el Sex linguarum... dilucidissimus dictionarius de Philipp Ulhart", in Pecia Complutense. Año 4. Num. 7. pp. 29-43

Pluche, Abbé (1751). La mécanique des langues. Paris: Chez la veuve Estienne\&Fils.

Puren, Ch. (1988). Histoire des méthodologies de l'enseignement des langues. Paris : Nathan.

Rossebastiano Bart, A. (ed.) (1984). Antichi vocabolari plurilingui d'uso popolare: la tradizione del 'Solenissimo Vochabuolista'. Alessandri: Edizionidell'Orso.

Sáez Rivera, D. M. (2004). "La explotación pedagógica del diálogo escolar en la didáctica del español (ss. XVI-XIX)", in ESELE. Actas XV, 792-797.

Sánchez Pérez, A. (1992). Historia de la enseñanza del español. Madrid: SGEL. 